\title{
RETRATIBILIDADE E MASSA ESPECÍFICA DA MADEIRA DE Inga marginata Willd.
}

\section{Luana Candaten $^{1}$ Alcir Vianei Scheeren Klauck ${ }^{1}$ Eduarda Bandera $^{1}$ Tauana de Souza Mangini ${ }^{1}$ Rômulo Trevisan ${ }^{2}$}

${ }^{1}$ Graduandos em Engenharia Florestal pela Universidade Federal de Santa Maria - Campus

Frederico Westphalen, e-mail: luana_candaten@outlook.com.

${ }^{2}$ Professor Doutor da Universidade Federal de Santa Maria - Campus Frederico

Westphalen.

Recebido em: 22/09/2018 - Aprovado em: 23/11/2018 - Publicado em: 03/12/2018 DOI: $10.18677 /$ EnciBio_2018B71

\section{RESUMO}

Os estudos com espécies nativas são fundamentais, pois podem auxiliar na caracterização e utilização desse material nos mais variados fins e, ainda, contribuir na difusão de novas tecnologias e informações sobre esses indivíduos arbóreos. Com isso, o presente trabalho teve por objetivo determinar a retratibilidade e a massa específica da madeira de Inga marginata Willd. Para tanto, foram abatidas três árvores com, aproximadamente, seis anos de idade, das quais foi retirada a primeira tora de cada uma dessas, para a determinação das propriedades físicas. As dimensões e a massa dos corpos de prova, na posição amostrada, foram mensuradas em duas condições de umidade (saturado e anidra) e as propriedades físicas foram determinadas conforme as recomendações da NBR 7190 (ABNT, 1997). A massa específica básica média na primeira tora dessa espécie foi de 0,487 $\mathrm{g} / \mathrm{cm}^{3}$, sendo caracterizada como média. Já a massa específica aparente média apresentou valores iguais a 0,561 e $1,152 \mathrm{~g} / \mathrm{cm}^{3}$ para as condições $0 \%$ de umidade e a saturada, respectivamente. Os dados aritméticos de contração obtidos foram de $5,7,5,6$ e $0,8 \%$ para os sentidos tangencial, radial e longitudinal, respectivamente. Com relação ao coeficiente anisotrópico foi possível classificar o material como excelente, pois o valor médio foi igual a 1,2.

PALAVRAS-CHAVE: condições de umidade, espécie nativa, propriedades físicas da madeira.

\section{RETRATIBILITY AND SPECIFIC DENSITY OF THE Inga marginata Willd WOOD}

\begin{abstract}
The studies about native species are fundamental since they can help in the characterization and use of these materials in a wide range of purposes, and also, they can contribute to the diffusion of new technologies and information on these arboreal individuals. Thus, the present work aimed to determine the retratibility and the specific density of the Inga marginata Willd wood. For that, three trees with approximately six years of age were cut, from which the first log was removed for the determination of physical properties. The dimensions and mass of the specimens were measured in two moisture conditions (saturated and anhydrous) and the physical properties were determined according to the recommendations of NBR 7190 (ABNT, 1997). The mean specific density in the first log for this species was $0.487 \mathrm{~g} /$
\end{abstract}


$\mathrm{cm} 3$, being characterized as very light. The mean apparent specific density presented values equal to 0.561 and $1.152 \mathrm{~g} / \mathrm{cm} 3$ for the $0 \%$ of humidity and the saturated conditions, respectively. The arithmetic contraction data obtained were 5.7, 5.6 and $0.8 \%$ for the tangential, radial and longitudinal directions, respectively. In relation to the anisotropic coefficient, it was possible to classify the material as excellent since the average value was equal to 1.2 .

KEYWORDS: moisture conditions, native species, physical properties of wood.

\section{INTRODUÇÃO}

De maneira geral, as espécies madeireiras nativas foram, por muitas décadas, amplamente exploradas pelo seu destaque como material de excelente qualidade para indústria de móveis, carpintaria, construção civil, naval e também utilizadas na medicina tradicional (RIBEIRO et al., 2015). Aliado ao baixo percentual de germinação das sementes, comum em espécies nativas (CARVALHO, 2014), estas decresceram numericamente suas populações e a variabilidade genética (LINGNER et al., 2015), porém em contrapartida, a demanda por essa matéria-prima apresentou aumento nos últimos anos (SOHNGEN; TIAN, 2016).

Com isso, o estudo das características tecnológicas da madeira proveniente de florestas nativas torna-se importante uma vez que o conhecimento, entendimento e difusão das informações encontradas poderão contribuir para o correto emprego desse material nos mais variados fins. Dentre essas, o Inga maginata Willd., também conhecido como Ingá-feijão, é um exemplo de espécie arbórea nativa do estado do Rio Grande do Sul, com ocorrência natural em várias regiões do Brasil e também no nordeste da Argentina destacando-se em ambientes úmidos (GARCIA; FERNANDES, 2015).

Entretanto, a conceituação geral da madeira é importante para o melhor entendimento do estudo, sendo um material poroso, higroscópico, anisotrópico e heterogêneo que, aliado com a genética e as condições do ambiente em que está submetido, pode resultar em diferentes características e variações de ordem anatômica, física, química e de resistência mecânica (LOPES et al., 2011; MELESSE; ZEWOTIR, 2013).

Assim, a espécie pode apresentar determinadas peculiaridades de tal modo a restringir seu uso sob certas condições (ROCHA et al., 2015), tornando indispensável a avaliação de uma série de fatores (ARAÚJO et al., 2016) como, por exemplo, as propriedades físicas. Essas características variam entre espécies, indivíduos da mesma espécie e, ainda, dentro de um único fuste no sentido medulacasca e da base para o topo (DIAS JÚNIOR et al., 2013; RIBEIRO et al., 2013; MELO et al., 2014), como o caso de elevada densidade pode acarretar numa maior resistência mecânica podendo ser ideal esse material para a construção civil, e madeiras com alto coeficiente anisotrópico resulta numa menor empregabilidade dessa espécie por esse parâmetro ser negativo na qualidade do material.

Nesse sentido, a massa específica é uma das mais importantes propriedades físicas a ser definida, uma vez que está intimamente associada com outras características tecnológicas (CHAGAS, 2014; LIMA et al., 2014; TREVISAN et al., 2016) e pode ser empregada como uma indicadora paramétrica de qualidade da madeira (TRAUTENMÜLLER et al., 2014).

Outro parâmetro que pode ser levado em consideração na avaliação e classificação do material é a sua retratibilidade, ou seja, a resposta da madeira a determinados ambientes aos quais é exposta por consequência das condições de 
umidade que, em função de sua higroscopicidade, pode resultar em variações dimensionais quando há alteração no teor de umidade abaixo do ponto de saturação das fibras (PSF) (OLIVEIRA,1990). De maneira geral, isso ocorre porque moléculas de água estão ligadas por meio de pontes de hidrogênio às microfibrilas dos polissacarídeos que formam a madeira, podendo variar de acordo com a espécie (OLIVEIRA et al., 2010).

Assim, a determinação das propriedades físicas do material torna-se importante na destinação final e, dessa forma, o objetivo do presente trabalho foi avaliar a retratibilidade e massa específica da madeira de Inga marginata Willd.

\section{MATERIAL E MÉTODOS}

O material utilizado no presente estudo foi amostrado na área pertencente à Universidade Federal de Santa Maria - Campus de Frederico Westphalen, onde três árvores de Inga marginata Willd. com, aproximadamente, seis anos de idade foram abatidas e a primeira tora na posição base-topo com 1,40 m de comprimento foi selecionada de cada indivíduo para a determinação das características físicas da madeira.

O desdobro desse material foi realizado com o auxílio de uma serra fita adotando o sistema de cortes tangenciais para confecção de duas tábuas por indivíduo com dimensões de $1000 \mathrm{~mm} \times 50 \mathrm{~mm} \times 10 \mathrm{~mm}$ (comprimento, largura e espessura, respectivamente) e posteriormente confeccionados 16 corpos de prova por tábua com as dimensões sugeridas na norma NBR 7190 (1997). Concluída essa etapa, as peças foram transportadas para o Laboratório de Tecnologia e Utilização de Produtos Florestais da instituição onde foram retirados dezesseis corpos de prova para determinação do teor de umidade saturado (inicial), massa específica básica, massa específica aparente ao teor de umidade saturado (inicial) e seca na condição anidra e da retratibilidade (contração e coeficiente anisotrópico) da madeira de acordo com a NBR 7190 (ABNT, 1997).

Para a análise do teor de umidade saturado os corpos de prova foram, de forma imediata, primeiramente pesados em balança com precisão de $0,01 \mathrm{~g}$. Após essa mensuração, os mesmos foram encaminhados para secagem em estufa à $103^{\circ} \mathrm{C}$, com seus pesos acompanhados periodicamente, até alcançarem valor constante, ou seja, até atingirem a condição anidra (Equação 1).

$$
\mathrm{Tu}=\frac{\mathrm{Pu}-\mathrm{P}_{0 \%}}{\mathrm{P}_{0 \%}} \times 100
$$

Onde: $\mathrm{Tu}=$ Teor de umidade saturado (\%); $\mathrm{P}_{\mathrm{u}}=$ Peso saturado $(\mathrm{g}) ; \mathrm{P}_{0 \%}=$ peso seco a $0 \%$ de umidade $(\mathrm{g})$.

O volume das amostras para as determinações das massas específicas básica (Equação 2), aparente saturada e na condição anidra (Equação 3) foi realizado pelo método da balança hidrostática. Já o peso, nas respectivas umidades, foi obtido pelo método gravimétrico em balança analítica com precisão de $0,01 \mathrm{~g}$.

$$
\mathrm{Me}_{\text {Básica }}=\frac{\mathrm{P}_{0 \%}}{\mathrm{~V}_{\mathrm{V}}}
$$


Onde: $\mathrm{Me}_{\text {Básica }}=$ Massa específica básica $\left(\mathrm{g} / \mathrm{cm}^{3}\right) ; \mathrm{P}_{0 \%}=$ peso seco a $0 \%(\mathrm{~g}) ; \mathrm{V}_{\mathrm{V}}=$ Volume verde $\left(\mathrm{cm}^{3}\right)$.

$$
\mathrm{Me}_{\text {Aparente } \mathrm{xi}}=\frac{\mathrm{Pxi}}{\mathrm{Vxi}}
$$

Onde: $\mathrm{Me}_{\text {Aparente xi }}=$ Massa específica aparente na condição de umidade "xi" $\left(\mathrm{g} / \mathrm{cm}^{3}\right)$; $\mathrm{P}=$ Peso na condição de umidade " $\mathrm{xi}$ " $(\mathrm{g}) ; \mathrm{V}_{\mathrm{x}}=$ Volume na condição de umidade "xi" $\left(\mathrm{cm}^{3}\right)$; $\mathrm{x} i=$ condição de umidade (onde $\mathrm{i}=1$ para madeira saturada $\mathrm{e} \mathrm{i}=2$ para material anidro).

Para a determinação da contração (Equação 4) os corpos de prova foram mensurados em pontos previamente demarcados nos sentidos radial, tangencial e longitudinal utilizando um paquímetro com precisão de $0,01 \mathrm{~mm}$, nas condições de umidade saturada e anidra, de acordo com a NBR 7190 (ABNT, 1997).

$$
\beta_{(r, t, l)}=\frac{D v-D s}{D v} \times 100
$$

Onde: $\beta(r, t, l)=$ contração nos sentidos radial, tangencial e longitudinal; (\%); $D v=$ dimensão saturada; ( $\mathrm{mm})$; e $\mathrm{Ds}=$ dimensão seca a $0 \%$ de umidade $(\mathrm{mm})$.

Os dados coletados das variáveis em estudo foram submetidos a uma análise descritiva utilizando o programa de computador Statgraphics.

\section{RESULTADOS E DISCUSSÃO}

Os valores obtidos nos ensaios das propriedades físicas da madeira de Inga marginata são encontrados na Tabela 1, onde é possível observar os dados para cada indivíduo amostrado e também a média geral.

TABELA 1 - Valores médios do teor de umidade saturado, massa específica básica e aparente seco a $0 \%$ de umidade e saturada, contração (sentidos tangencial, radial e longitudinal) e coeficiente anisotrópico da madeira de Inga marginata.

\begin{tabular}{ccccc}
\hline Propriedades & Árvore 1 & Árvore 2 & Árvore 3 & Média geral \\
\hline TU $(\%)$ & 140,0 & 128,1 & 144,8 & 137,6 \\
& $( \pm 14,3)$ & $( \pm 12,5)$ & $( \pm 8,2)$ & $( \pm 13,6)$ \\
MEb $\left(\mathrm{g} / \mathrm{cm}^{3}\right)$ & 0,488 & 0,507 & 0,465 & 0,487 \\
& $( \pm 0,045)$ & $( \pm 0,038)$ & $( \pm 0,017)$ & $( \pm 0,031)$ \\
MEa 0\% $\left(\mathrm{g} / \mathrm{cm}^{3}\right)$ & 0,558 & 0,593 & 0,530 & 0,561 \\
& $( \pm 4,3)$ & $( \pm 4,3)$ & $( \pm 2,1)$ & $( \pm 3,6)$ \\
MEa saturada $\left(\mathrm{g} / \mathrm{cm}^{3}\right)$ & 1,166 & 1,153 & 1,138 & 1,152 \\
& $( \pm 0,043)$ & $( \pm 0,036)$ & $( \pm 0,013)$ & $( \pm 0,031)$ \\
$\beta$ tangencial $(\%)$ & 5,6 & 5,8 & 5,7 & 5,7 \\
ß radial $(\%)$ & $( \pm 1,5)$ & $( \pm 1,6)$ & $( \pm 1,0)$ & $( \pm 1,4)$ \\
& 5,2 & 5,1 & 6,2 & 5,6 \\
$\beta$ longitudinal $(\%)$ & $( \pm 1,6)$ & $( \pm 1,1)$ & $( \pm 1,1)$ & $( \pm 1,3)$ \\
& 0,6 & 1,1 & 0,8 & 0,8 \\
CA & $( \pm 0,3)$ & $( \pm 0,4)$ & $( \pm 0,3)$ & $( \pm 0,3)$ \\
& 1,4 & 1,1 & 1,0 & 1,2 \\
& $( \pm 0,3)$ & $( \pm 0,4)$ & $( \pm 0,3)$ & $( \pm 0,3)$ \\
\hline
\end{tabular}


Onde: $\mathrm{Tu}=$ teor de umidade; $\mathrm{MEb}=$ massa específica básica; $\mathrm{MEa} 0 \%$ = massa específica aparente seco a $0 \%$; MEa saturada = massa específica aparente saturada; $\beta$ tangencial $=$ contração no sentido tangencial; $\beta$ radial $=$ contração no sentido radial; $\beta$ longitudinal $=$ contração no sentido longitudinal; $\mathrm{CA}=$ coeficiente anisotrópico. Valores entre parênteses correspondem ao desvio padrão.

O teor de umidade obtido a partir do material saturado foi semelhante ao observado por Pertuzzatti et al. (2017) para a madeira de Eucalyptus grandis com valor igual a $151,8 \%$ e mostra-se menor que o da espécie Eucalyptus cloeziana sendo de $80,4 \%$ também estudada pelos mesmos autores. Essa variação dos resultados entre as árvores caracterizadas como folhosas pode ter ocorrido em função da capacidade de absorver água que é desigual entre as diferentes espécies.

De maneira geral as árvores apresentaram valores médios de massa específica básica da madeira próximos aos encontrados na literatura e com uma amplitude de 0,465 a $0,507 \mathrm{~g} / \mathrm{cm}^{3}$. Isso pode ser evidenciado no estudo realizado para a mesma espécie por Trautenmüller et al. (2016), onde esta propriedade tecnológica variou de 0,487 a $0,520 \mathrm{~g} / \mathrm{cm}^{3}$ sendo semelhante ao observado no presente trabalho. Ainda, Tomasi et al. (2013) obtiveram uma massa específica básica média igual a $0,510 \mathrm{~g} / \mathrm{cm}^{3}$ para Ateleia glazioviana, espécie da mesma família do Inga marginata. Essas variações nos dados podem ser entendidas em função dos fatores químicos, físicos e anatômicos que são influenciados por genótipo, variações ambientais, idade da árvore, influências externas e até pela determinação em diferentes posições do tronco das árvores (DELUCIS et al., 2013; ZAQUE et al., 2018). De acordo com a classificação de Melo et al. (1992) essa madeira enquadrase como média em função de sua massa específica básica estar na amplitude de $0,460 \mathrm{~g} / \mathrm{cm}^{3}$ a $0,540 \mathrm{~g} / \mathrm{cm}^{3}$.

Os resultados de massa específica aparente na condição saturada e a $0 \%$ de umidade apresentaram valores que corroboraram com outras arbóreas folhosas como, por exemplo, o Eucalyptus grandis, espécie amplamente utilizada nos mais variados segmentos, conforme observado por Pertuzzatti et al. (2017). Os mesmos autores abordaram, ainda, que a massa específica aparente saturada está relacionada com a saturação total das fibras que, acima do PSF, aumenta somente o peso do material resultando em maiores valores dessa propriedade conforme aumenta o teor de umidade.

Conforme apresentado na Tabela 1, as alterações dimensionais (contração) foram semelhantes nos sentidos tangencial e radial, sendo praticamente desprezíveis no longitudinal. De maneira geral, sabe-se que a contração tangencial pode ser o dobro da radial e cerca de 20 vezes maior que no sentido longitudinal (DURLO; MARCHIORI, 1992), resultados não observados na presente análise.

Dentre essas, Tomasi et al. (2013) para madeira de Ateleia glazioviana encontraram valores de contração tangencial e radial, menores e maiores, respectivamente, quando comparado com os obtidos no presente estudo. Em relação ao coeficiente anisotrópico esse se mostrou expressivamente superior, apresentando valores próximos a 2. Ainda, as propriedades de contração e anisotropia foram inferiores ao observado no trabalho de Costa et al. (2017) para Mimosa schomburgkii, que variaram de 7,8 a 12,1\% para a posição tangencial e 5,0 a $7,0 \%$ para radial. Já o coeficiente anisotrópico também apresentou valores ligeiramente mais elevados e os autores abordaram que quanto mais elevado esse parâmetro, mais propenso o material está a ocorrência de defeitos como o fendilhamento e empenamento ocasionados pela alteração dimensional a qual a ENCICLOPÉDIA BIOSFERA, Centro Científico Conhecer - Goiânia, v.15 n.28; p.882 2018 
anisotropia se refere. Com isso, os valores estimados no presente trabalho são desejáveis para a madeira e determinantes para o seu beneficiamento.

\section{CONCLUSÕES}

Os resultados indicam que a madeira de Inga marginata Willd. é considerada média em função da massa específica.

As alterações dimensionais apresentam valores positivos para o uso da madeira da espécie nas mais variadas funções como construção civil, fabricação de móveis e demais produtos madeireiros.

\section{REFERÊNCIAS}

ABNT - Associação Brasileira de Normas Técnicas. NBR 7190/97: Estruturas de madeira. Rio de Janeiro, p. 107, 1997.

ARAÚJO, B. H. P.; SOUSA, M. A R.; NASCIMENTO, H. E. M; ZANUNCIO, A. J. V.; RODRIGUES, D. M. S.; et al. Propriedades físicas da madeira de Calycophyllum spruceanum Benth. em função do diâmetro e da posição (base e topo) no fuste. Scientia Forestalis, Piracicaba, v. 44, n. 111, p. 759-768, 2016. Disponível em: < http://www.ipef.br/publicacoes/scientia/nr111/cap22.pdf> dx.doi.org/10.18671/scifor.v44n111.22.

CARVALHO, P. E. R. Espécies arbóreas brasileiras. Colombo: Embrapa CNPF; Brasilia, D.F.: Embrapa-SPI, p. 633, 2014.

CHAGAS, S. F.; EVANGELISTA, W. V.; SILVA, J. de C.; CARVALHO, A. M. M. L. Propriedades da madeira de teca de diferentes idades e oriundas de desbaste. Ciência da Madeira, Pelotas, 2014. Disponível em: < https://periodicos.ufpel.edu.br/ojs2/index.php/cienciadamadeira/article/viewFile/4806/ 3578>. doi: 10.12953/2177-6830.v05n02a08.

COSTA, L. J.; LOPES, C. B. da S.; REIS, M. F. de C.; CANDIDO, W. L.; FARIA, B. de F. H.; et al. Caracterização anatômica e descrição físico-química e mecânica da madeira de Mimosa schomburgkii. Revista Floresta, Curitiba, v. 47, n. 4, p. 383-390, 2017. Disponível em: < https://revistas.ufpr.br/floresta/article/view/54471/34360>. doi: 10.5380/rf.v47i4.54471.

DELUCIS, R. A.; GATTO, D. A.; STANGERLIN, D. M.; BELTRAME, R.; TREVISAN, R. Qualificação da madeira de três espécies de coníferas oriundas de reflorestamentos jovens. Scientia Forestalis, Piracicaba, v. 41, n. 100, p. 477-484, 2013.

em:

<https://periodicos.ufpel.edu.br/ojs2/index.php/cienciadamadeira/article/view/4132>.

DIAS, A. F. J.; SANTOS, P. V.; PACE, J. H. C.; CARVALHO, A. M.; LATORRACA, J. V. F. Caracterização da madeira de quatro espécies florestais para uso em movelaria. Ciência da Madeira, Pelotas, v. 4, n. 1, p. 93-107, 2013. Disponível em: $<$ https://periodicos.ufpel.edu.br/ojs2/index.php/cienciadamadeira/article/view/4048/0> doi: 10.12953/2177-6830.v04n01a08 
DURLO, M. A.; MARCHIORI, J. N. C. Tecnologia da madeira: retratibilidade. Santa Maria: CEPEF/FATEC, p. 33, 1992. (Série técnica, 10).

GARCIA, F. C. P.; FERNANDES, J. M. Lista de Espécies da Flora do Brasil, 2015. Jardim Botânico do Rio de Janeiro. Disponível em: $<$ http://floradobrasil.jbrj.gov.br/jabot/floradobrasil/FB23000> .

LIMA, I. L.; LONGUI, E. L.; FREITAS, M. L. M.; ZANATTO, A. C. S.; ZANATA, M.; et al. Physical-mechanical and anatomical characterization in 26-year-old Eucalyptus resinifera wood. Floresta e Ambiente, Seropédica, v. 21, n. 1, p. 91-98, 2014. Disponível em: < http://www.scielo.br/pdf/floram/v21n1/aop_floram_055513.pdf>. doi: 10.4322/floram.2014.006.

LINGNER, D. V.; SCHORN, L. A.; SEVEGNANI, L.; GASPER, A. L.; MEYER, L.; et al. Floresta Ombrófila Densa de Santa Catarina - Brasil: Agrupamento e ordenação baseados em amostragem sistemática. Ciência Florestal, Santa Maria, v. 25, n. 4, p. 933-946, 2015. Disponível em: <http://www.scielo.br/pdf/cflo/v25n4/0103-9954cflo-25-04-00933.pdf> doi: 10.5902/1980509820595.

LOPES, C. S. D.; NOLASCO, A. M.; TOMAZELLO, M. F.; DIAS, C. T. S.; PANSINI, A. Estudo da massa específica básica e da variação dimensional da madeira de três espécies de eucalipto para a indústria moveleira. Ciência Florestal, Santa Maria, v. 21, n. 2, p. 315-322, 2011. Disponível em: <http://www.bioline.org.br/pdf?cf11033>.

MELESSE, S. F.; ZEWOTIR, T. The effect of correlated climatic factors on the radial growth of eucalypt trees grown in coastal Zululand of South Africa. African Journal of Agricultural Research, Nigéria, v. 8, n. 14, p. 1233-1244, 2013. Disponível em: <http://academicjournals.org/journal/AJAR/article-full-text-pdf/0B6FE4934981> doi: 10.5897/AJAR12.1671.

MELO, J. E.; CORADIN, V. T. R.; MENDES, J. C. Classes de densidade para madeiras da Amazônia brasileira. Silvicultura, São Paulo, v. 12, n. 42, p. 695-699, 1992.

em: <http://www.eucalyptus.com.br/eucaexpert/1037_Classificacao\%20madeiras\%20euc alipto\%20pela\%20densidade.pdf>.

MELO, L. E. L.; SILVA, C. J.; PROTÁSIO, T. P.; TRUGILHO, P. F.; SANTTOS, I. S.; et al. Influence of spacing on some physical properties of Schizolobium parahyba var. amazonicum (Huber ex Ducke). Scientia Forestalis, Piracicaba, v. 42, n. 104, p. 483-490, $2014 . \quad$ Disponível em: <http://www.ipef.br/publicacoes/scientia/nr104/cap02.pdf>.

OLIVEIRA, E. Correlações entre parâmetros de qualidade da madeira de Eucalyptus grandis Hill ex-Maiden, Boletim Técnico da SIF, Viçosa, n. 2, p. 1-9, 1990.

OLIVEIRA, J. T. da S.; TOMAZELLO FILHO, M.; FIEDLER, N. S. Avaliação da retratibilidade da madeira de sete espécies de Eucalyptus. Revista Árvore, Viçosa, v. 34, n. 5, p. 929-936, 2010. Disponível em: < http://www.scielo.br/pdf/rarv/v34n5/18.pdf>. 
PERTUZZATTI, A.; CONTE, B.; MISSIO, A. L. M.; GATTO, D. A.; HASELEIN, C. R. et al. Influência da umidade na resistência da madeira de eucalipto a impactos. Floram, Seropédica, v. 24, p. 1-6, 2017. Disponível em: < https://www.researchgate.net/profile/Andre_Missio2/publication/318871262_Influenci a_da_Umidade_na_Resistencia_da_Madeira_de_Eucalipto_a_Impactos/links/5984af 75a6fdcc75624fbec2/Influencia-da-Umidade-na-Resistencia-da-Madeira-deEucalipto-a-Impactos.pdf>. doi: doi.org/10.1590/2179-8087.092514.

RIBEIRO, P. G.; GONÇALEZ, J. C.; GONÇALVES, R.; TELES, R. F.; TELES, R. F.; et al. Ultrasound waves for assessing the technological properties of Pinus caribaea var hondurensis and Eucalyptus grandis wood. Maderas: Ciencia y Tecnología (En línea), Concepción, v. 15, p. 2-10, 2013. Disponível em: < http://www.revistamaderas.cl/>. doi: 10.4067/S0718-221X2013005000016.

RIBEIRO, E. F.; FÁTIMA, R. C.; CARVALHO, F. S.; ABREU, J. P.; ARRUDA, A. F. et al. Diuretic effects and urinary electrolyte excretion induced by Aspidosperma subincanum Mart. and the involvement of prostaglandins in such effects. Journal of Ethnopharmacology, Limerick, v. 163, p. 142-148, 2015. Disponível em: <https://www.ncbi.nlm.nih.gov/pubmed/25625354> doi: 10.1016/j.jep.2015.01.023

ROCHA, H. L. S.; PAES, J. B.; MINÁ, A. J. S; OLIVEIRA, E. Caracterização físico mecânica da madeira de jurema -preta (Mimosa tenuiflora) visando seu emprego na indústria moveleira. Ciências Agrárias, Recife, v. 10, n. 2, p. 262-267, 2015. Disponível em: <http://www.redalyc.org/pdf/1190/119039562015.pdf> doi: 10.5039/agraria.v10i2a3772.

SOHNGEN, B.; TIAN, X. 2016. Global climate change impacts on forests and markets. Forest Policy and Economics, Bangladesh, v. 72, p. 18-26, 2016. Disponível em: <https://ac.els-cdn.com/S1389934116301307/1-s2.0S1389934116301307 main.pdf? tid=ab32cbdf-0f86-4afb-a3a3 198b1300a88b\&acdnat $=153493747929 f 39 e c 7 f 2169$ cbf0211ccbba8649169> doi: org/10.1016/j.forpol.2016.06.06.011.

TOMASI, J. de C.; TRAMONTINA, J.; TRES, J.; CHECHI, L.; TREVISAN, R. Propriedades físicas da madeira de Ateleia glazioviana Baill. Enciclopédia biosfera, Centro Científico Conhecer, Goiânia, v.9, n.16, p.1824-1832, 2013. Disponível em: < http://www.conhecer.org.br/enciclop/2013a/agrarias/propriedades\%20fisicas.pdf>.

TRAUTENMÜLLER, J. W.; BALBINOT, R.; BORELLA, J.; TREVISAN, R.; BALESTRIN, D. et al. Variação longitudinal da massa específica básica da madeira de Cordia americana e Alchornea triplinervia. Ciência Rural, Santa Maria, v. 44, n. 5, p. $\quad 817-\quad 821, \quad 2014 . \quad$ Disponível em: <http://www.redalyc.org/html/331/33130634009>.

TRAUTENMÜLLER, J, W.; BORELLA, J.; BALBINOT, R.; LAMBRECHT, F. R.; VALERIUS, J. et al. Longitudinal variation of wood basic density of Inga marginata and Chrysophyllum gonocarpum. Advances in Forestry Science, Cuiaba, v. 3, n. 3, 
p. 2016. 45-49, 20 Disponível em: < http://periodicoscientificos.ufmt.br/ojs/index.php/afor/article/download/3609/pdf>.

TREVISAN, R.; ZANELLA, A.; SILVA, F. M. da.; ROSA, M.; FIORESI, T. et al. Axial variation of basic density of Araucaria angustifolia wood in different diameter classes. Ciência Rural, Santa Maria, v. 46, n. 11, p. 1969-1972, 2016. Disponível em: <http://www.scielo.br/pdf/cr/v46n11/1678-4596-cr-0103_8478cr20141312.pdf>. doi: 10.1590/0103-8478cr20141312.

ZAQUE, L. A. M.; FERREIRA, M. D.; MELO, R. R. Variação radial e longitudinal da massa específica básica da madeira de Araucaria angustifolia com diferentes idades. Pesquisa Florestal Brasileira, Colombo, v. 38, p. 1-5, 2018. Disponível em: <https://pfb.cnpf.embrapa.br/pfb/index.php/pfb/article/view/e01368/663>. doi: 10.4336/2018.pfb.38e201601368. 